\title{
Recent advances in type 1 diabetes
}

\begin{abstract}
“Нypo-
glycaemia

is a common

and often a

feared adverse

event that is

a major barrier

to attaining

near-normal

blood glucose

levels"

$\mathrm{T}$ ype 1 diabetes (T1D) affects around 120000 Australians, half of whom are diagnosed in adulthood. ${ }^{1}$ It is caused by the immune-mediated destruction of pancreatic beta cells, leading to insulin deficiency, hyperglycaemia and the risk of ketoacidosis. Antibodies directed against the beta-cell antigens insulin, glutamic acid decarboxylase 65 (GAD65), insulinomaassociated protein 2 (IA-2) and zinc transporter 8 (ZnT8) are markers of T1D autoimmunity used to confirm the diagnosis of T1D and to identify normoglycaemic people at high risk of progressing to T1D. ${ }^{2}$ The incidence of T1D has doubled in Australia during the past 20 years, ${ }^{3}$ leading to speculation that it is caused by environmental or epigenetic factors. Environmental changes that may play a pathogenic role include viral infections, a more hygienic environment, and increased caloric intake with associated weight gain. ${ }^{4}$
\end{abstract}

Mervyn Kyi

MBBS(Hons), BMedSCi

John M Wentworth $M B B S$, PhD, FRACP

Alison J Nankervis

MBBS, MD, FRACP

Spiros Fourlanos

MBBS, PhD, FRACP

Peter G Colman

MBBS, MD, FRACP

Royal Melbourne Hospital, Melbourne, VIC.

peter.colman@ mh.org.au

doi: 10.5694/mjal4.01691

Some individuals with insulin deficiency presenting as T1D do not express the typical beta-cell autoantibodies, and alternative diagnoses, such as early-onset type 2 diabetes or idiopathic (type 1b) diabetes, should be considered. Another possibility is monogenic diabetes, particularly in children with a strong family history and unusual clinical features, such as renal impairment or exocrine pancreatic insufficiency.

\section{General management principles}

Since the 1990s, the most common treatment strategy has been the combination of once- or twice-daily injections of long-acting insulin (eg, insulin detemir or glargine) and short-acting insulin (eg, neutral insulin or the aspart, lispro and glulisine insulin analogues) taken with meals. An individual's required dose of long-acting (basal) insulin is relatively constant, but can vary with exercise and physiological stressors, such as illness. Glargine and detemir insulin are commonly preferred to NPH (neutral protamine Hagedorn) insulin because their use is associated with lower rates of nocturnal and severe hypoglycaemia. Detemir is also associated with less weight gain than insulin NPH. All three long-acting insulins achieve similar reductions in glycated haemoglobin $\left(\mathrm{HbA}_{1 \mathrm{c}}\right)$ levels. ${ }^{5}$ Novel ultralong-acting insulin analogues are being developed. Insulin degludec provides basal insulin coverage for more than 40 hours, and achieves similar glycaemic control with less overnight hypoglycaemia than glargine. ${ }^{6}$ Degludec is approved for use in Europe, but is yet to be approved by the United States Food and Drug Administration or the Australian Therapeutic Goods Administration.

Bolus (meal-time) insulin requirements in T1D are more variable than basal insulin requirements, depending primarily on carbohydrate intake. Accordingly, many people with T1D learn to determine the carbohydrate content of their meals ("carbohydrate counting") to guide their prandial insulin dose. Studies of advanced carbohydrate

\section{Summary}

- Type 1 diabetes (TID) is caused by an autoimmune attack on pancreatic beta cells that leads to insulin deficiency.

- The incidence of TID in Australia has doubled over the past 20 years.

- TID treatment focuses on physiological insulin replacement, aiming for near-normal blood glucose levels.

- Hypoglycaemia is a significant cause of morbidity and mortality in TID.

- Optimal TID management is complex, and is enhanced by empowering individuals in all aspects of managing diabetes.

- New technologies, including insulin pumps, continuous glucose monitors and sensor-augmented pumps, can assist people achieve better glycaemic control and reduce the risk of severe hypoglycaemia.

- Women with TID can achieve significantly better outcomes during pregnancy and for their infants by planning for their pregnancy and by intensive glycaemic control.

- Several trials are underway that seek to identify the determinants of autoimmunity and to develop therapies that prevent TID in at-risk individuals.

- Pancreatic and islet cell transplants are proven therapies, but are only offered to individuals with diabetes and renal failure (pancreas) or severe hypoglycaemia unawareness (islet cell transplants).

- Although TID is still associated with considerable premature mortality, recent findings show that a significant improvement in life expectancy has occurred.

counting in T1D suggest that it reduces $\mathrm{HbA}_{1 \mathrm{c}}$ levels and the frequency of hypoglycaemia.?

Ideally, people with T1D learn to self-adjust their bolus insulin dose to achieve optimal glycaemic control, using calculations based on personal experience and clinical observation. The specific formulas, incorporating the insulin-to-carbohydrate ratio and the insulin sensitivity factor, are provided in the Box. Self-adjustment of insulin dose can be learned informally or during structured training courses, such as the Dose Adjustment for Normal Eating (DAFNE) program (www.dafne.org.au). ${ }^{8}$ Central to self-adjustment of insulin dose is the self-monitoring of blood glucose levels. This requires a minimum of four glucose checks each day: once before each meal, and at bedtime. Glucose checks 2 hours after a meal can also be used to assess the adequacy of the bolus insulin dose for the preceding meal.

Variability in both insulin absorption after subcutaneous injection and of carbohydrate absorption from the gut can contribute to glycaemic variation in T1D. Areas of lipohypertrophy that can result from repeated insulin 
Bolus insulin self-titration calculation

- Carbohydrate ratio (CHR): grams of carbohydrate covered by 1 unit of insulin.

- Insulin sensitivity factor (ISF): drop in blood glucose level (in mmol/L) achieved by each unit of insulin.

Sample calculation:

- Tim has TID. His CHR is $10: 1$ and his ISF is $2: 1$.

- He is about to have a lunch that includes $50 \mathrm{~g}$ of carbohydrate.

- His current blood glucose level (BGL) is $14 \mathrm{mmol} / \mathrm{L}$. His target BGL is $6 \mathrm{mmol} / \mathrm{L}$.

- Prandial insulin dose $=$ carbohydrate load $\div \mathrm{CHR}$ or $50 \mathrm{~g} \div 10=5$ units of insulin to cover the carbohydrate in the meal.

- Correctional insulin dose $=$ variance from target glucose $\div$ ISF or $(14-6) \div 2=4$ units of insulin to correct BGL from $14 \mathrm{mmol} / \mathrm{L}$ to $6 \mathrm{mmol} / \mathrm{L}$.

- He should therefore take 9 units of short-acting insulin with his meal.

injections at the same site may further impair insulin absorption. Development of lipohypertrophy is less likely if the injection site is rotated regularly.

Exercise and illness are also potent modifiers of glycaemia. The acute glycaemic effects of exercise can vary according to the intensity of physical activity, but there is generally a tendency to delayed hypoglycaemia and a reduced insulin requirement.

All individuals should have a thorough knowledge of sick-day management, including more frequent glucose monitoring, ketone testing, self-adjustment of insulin dose, maintaining oral intake of food and liquid, and having a low threshold for seeking medical attention.

As with any chronic disease, psychosocial factors strongly influence T1D self-management. An individual may fluctuate between active management and total disengagement, depending on social circumstances and psychological factors, such as anxiety and depression. Health care providers should recognise these challenges. Taking social factors into account, referring people with T1D to appropriate psychological services, and gently helping the individual re-engage with self-care usually achieves better outcomes than focusing only on medical management.

Hypoglycaemia is a common and often a feared adverse event that is a major barrier to attaining near-normal blood glucose levels. Adults with T1D experience, on average, two mild hypoglycaemic episodes each week. More severe hypoglycaemia (requiring external assistance for recovery) occurs with an annual prevalence of $30 \%$. ${ }^{9}$ The risk of hypoglycaemia increases with the duration of disease and is inversely correlated with $\mathrm{HbA}_{1 \mathrm{c}}$ levels. Hypoglycaemia is a recognised cause of seizure and coma, and recent evidence suggests it may also cause serious cardiac ischaemia and arrhythmia. In addition, recurrent severe hypoglycaemia in children with earlyonset T1D is associated with lower cognitive test scores in adulthood. ${ }^{9}$ Severe hypoglycaemia and hypoglycaemic unawareness also underlie diabetes-related distress and poorer emotional well-being. ${ }^{10}$ Glycaemic targets should therefore be individualised, and must always be balanced against the risk of hypoglycaemia. It is encouraging that the incidence of severe hypoglycaemia in a cohort of Australian children with T1D has decreased over the past decade from 17.3 to 5.8 per 100 patient-years. $^{11}$

\section{New technologies that assist with glucose control}

There has been an explosion in the availability of new technologies that assist the self-management of T1D. New blood glucose meters are more convenient than older models, with streamlined electronic data upload and connectivity to health care teams. Blood glucose meters that include a built-in bolus dose calculator (also called "smart meters") integrate the insulin-to-carbohydrate ratio and insulin sensitivity factor when calculating insulin doses for each meal. There is, however, no strong evidence for improved glucose control in individuals using these devices. ${ }^{12}$ Several smartphone applications are now available to assist with various aspects of selfmanagement, including carbohydrate counting, glucose monitoring and insulin dose calculations.

Insulin pumps are becoming increasingly popular, and are used by about $10 \%$ of Australians with T1D. ${ }^{13}$ Insulin pumps deliver short-acting insulin through a subcutaneous cannula that the patient re-positions every 3 days. Insulin is delivered continuously, and the rate can be varied to better mimic endogenous insulin production and to match physical activity. Prandial and correctional insulin boluses are administered via the pump under the control of the user. Compared with multidose insulin therapy, pump therapy is associated with a reduction in $\mathrm{HbA}_{1 \mathrm{c}}$ levels of about $3.3 \mathrm{mmol} / \mathrm{mol}$ $(0.3 \%)$, but, more importantly, also with reduced rates of severe hypoglycaemia and improved quality of life. ${ }^{14}$ However, their safe and successful operation requires the close engagement of the individual with their pump, their willingness to undertake adequate glucose monitoring, and their proficiency in carbohydrate counting. Insulin pumps cost up to $\$ 9500$, which can generally be partially or fully reimbursed by private health insurers; the government provides a subsidy for children from low-income families.

Continuous glucose monitoring systems measure interstitial fluid glucose concentrations every 5 minutes via a thin glucose-sensing subcutaneous cannula. Continuous glucose monitors can be used in individuals with T1D for a week at a time to retrospectively assess the 24-hour blood glucose profile and to diagnose unrecognised hypo- and hyperglycaemia. They can also be employed for longer periods, together with an insulin pump, to provide real-time glucose readings. These sensoraugmented pumps enable individuals to adjust insulin doses in response to glucose level trends, and to safely reduce $\mathrm{HbA}_{1 \mathrm{c}}$ levels by about $3.3 \mathrm{mmol} / \mathrm{mol}(0.3 \%) .{ }^{15}$ Sensor-augmented pumps also incorporate a "lowglucose-suspend" feature that temporarily stops insulin 
infusion if the blood glucose level is moving towards the hypoglycaemic range. ${ }^{16}$

Initial trials of closed-loop insulin systems (the so-called "artificial pancreas") that combine a continuous glucose monitor, a pump delivering insulin alone or in combination with glucagon, and a computer algorithm that determines the insulin dose, suggest that this strategy will deliver further improvements in glucose control. ${ }^{17}$ These systems are still in development, and are currently available in Australia only through participation in a clinical trial.

\section{Non-insulin treatments that assist with glucose control}

In type 2 diabetes (T2D), metformin is used to reduce the required insulin dose, prevent weight gain and reduce cardiovascular risk. More than a third of children and half of adults with T1D are overweight or obese, ${ }^{18,19}$ and a meta-analysis has shown that metformin therapy is associated with reduced levels of total cholesterol and low-density lipoprotein, ${ }^{20}$ suggesting that metformin might help prevent cardiovascular disease in T1D. This hypothesis is being tested in the REducing with Metformin Vascular Adverse Lesions in type 1 diabetes (REMOVAL) study currently underway in Europe, Canada and Australia (https://www.clinicaltrials.gov/ ct2/show/NCT01483560).

Other T2D medications are being tested for their ability to reduce blood glucose variability in T1D. Pilot trials in T1D populations have found that the glucagon-like peptide-1 analogue liraglutide suppresses glucagon levels and reduces insulin requirements, ${ }^{21}$ that the dipeptidyl peptidase-4 inhibitor sitagliptin showed a trend to reducing hyperglycaemia in a subset of patients, ${ }^{22}$ and that the sodium-glucose cotransporter 2 inhibitor dapagliflozin achieved a dose-related (but non-significant) reduction of glycaemic excursions and insulin requirements. $^{23}$ The use of these drugs in T1D is still experimental, pending further studies.

\section{Treatment targets and complications}

The importance of glucose control in T1D was shown by the landmark Diabetes Control and Complications Trial, ${ }^{24}$ which found that tighter glucose control $\left(\mathrm{HbA}_{1 \mathrm{c}}\right.$ level of $54 \mathrm{mmol} / \mathrm{mol}[7.1 \%]$ v $76 \mathrm{mmol} / \mathrm{mol}$ [9.1\%]) greatly diminished the risk of microvascular complications (retinopathy, nephropathy and neuropathy). Accordingly, the general $\mathrm{HbA}_{1 \mathrm{c}}$ target for adults is $\leq 53 \mathrm{mmol} / \mathrm{mol}$, although the target is lower in pregnancy (if this can be achieved safely) and less stringent for children and adolescents $(<58 \mathrm{mmol} / \mathrm{mol})$ and those prone to hypoglycaemia. ${ }^{25,26}$ The $\mathrm{HbA}_{1 \mathrm{c}}$ target also needs to be tailored to the clinical circumstances of each patient, including their disease duration, life expectancy and comorbidities. Screening for retinopathy (fundal examination), nephropathy (urine albumin/creatinine ratio) and neuropathy (clinical examination) should be performed at least annually, together with assessment of important cardiovascular risk factors, including smoking, blood pressure and lipids. Abnormalities should be treated according to national guidelines. ${ }^{26}$ Genetic predisposition appears to be an important determinant of nephropathy, although the relevant genes are yet to be identified.

\section{Pregnancy and TID}

Women with T1D can experience healthy pregnancies with excellent outcomes for their infants. However, optimal outcomes require effective preparation and excellent glycaemic control throughout the pregnancy; this generally requires support from an experienced diabetes-in-pregnancy team. Pregnancy preparation begins with educating young women with T1D about safe sexual practices (including contraception) and the need to plan for their pregnancy. All forms of contraception are safe for women with T1D, but hormonal contraception can adversely affect glucose control.

Preconception care includes optimising glucose control, assessing and treating diabetes complications, ensuring adequate folate $(2.5 \mathrm{mg}-5 \mathrm{mg}$ daily) and iodine $(150 \mu \mathrm{g}$ daily) intake, stopping or substituting potentially harmful medications, reviewing lifestyle factors (including diet, smoking and alcohol), and checking thyroid function, anti-transglutaminase antibodies for coeliac disease, and rubella and varicella immune status. Glucose control should be as close to target as is feasible. It is recommended that $\mathrm{HbA}_{1 \mathrm{c}}$ be $53 \mathrm{mmol} / \mathrm{mol}$ or less for 2-3 months prior to conception, and as low as is safely possible during the pregnancy. This reduces the risks of congenital malformations and miscarriage, the risk for each of which is roughly four times that for the general population. ${ }^{27,28}$ Insulin pumps can assist women achieve their glucose target, but similar control can be attained with an intensified multidose insulin regimen. Continuous glucose monitors are sometimes used to fine-tune management.

There is an increased risk of hypoglycaemia in early pregnancy, and hyperemesis may complicate glucose management. Insulin requirements usually increase substantially during the second half of pregnancy, when hyperglycaemia accelerates fetal growth. Hypertension, oedema, proteinuria and signs of evolving pre-eclampsia should be carefully monitored and treated aggressively, with consideration given to expediting delivery when clinically appropriate. If pregnancy is progressing well, delivery should be planned for around 38 weeks' gestation to reduce the risk of stillbirth and to minimise the risk of delivery complications. Although there have been significant improvements in neonatal outcomes, rates of macrosomia and caesarean delivery are higher for mothers with T1D than for the general population. ${ }^{28}$

After delivery, breastfeeding is encouraged. Insulin requirements change dramatically postpartum, and adjusting the insulin dose with the help of the health care team after discharge from hospital is essential. All outcomes are improved if management is undertaken at a 
tertiary centre with a specialist diabetes-in-pregnancy team and high-level neonatal care. ${ }^{29}$

\section{Prospects for the prevention of T1D}

The ability to predict T1D on the basis of genetic, immunological and metabolic markers has provided opportunities for prevention at different preclinical stages. Most attention has focused on interventions at diagnosis, a stage when significant amount of endogenous insulin is still produced. Treatment with anti-CD3, anti-CD20 or abatacept have shown clear promise and may be employed in the future to preserve beta-cell function. Trials are also underway investigating strategies that prevent T1D in high-risk populations characterised by detectable islet autoantibodies but with normal glucose tolerance. The intranasal insulin trial, led by an Australian team but including additional New Zealand and German sites, for instance, is examining whether a 12-month course of nasal insulin vaccine is an effective prevention strategy (https://clinicaltrials.gov/ct2/show/NCT00336674). An even more experimental strategy would be to prevent people with an elevated genetic risk from developing autoimmunity, a process that usually commences in utero and during the first few years of life. Central to this effort will be identifying the environmental triggers of autoimmunity, a question being investigated by the Environmental Determinants of Islet Autoimmunity Study.

\section{TID cure with pancreas or islet transplantation}

Whole pancreas transplant, usually performed simultaneously with a kidney transplant in people with T1D and renal failure, is a well-established therapy at the Westmead National Pancreas Transplant Unit in Sydney and the Monash Medical Centre in Melbourne. This procedure normalises blood glucose levels without requiring exogenous insulin, and also partially repairs established nephropathy, retinopathy, vascular disease and hypoglycaemic unawareness. Pancreatic graft survival is estimated to be greater than $60 \%$ at 5 years, but the benefits must be weighed against the morbidity associated with the surgery and associated immunosuppression. ${ }^{31}$

The concept of transplanting the islets of Langerhans to cure T1D was proposed more than 30 years ago, and remains an attractive but logistically difficult long-term solution. In Australia, islet isolation is undertaken at Westmead Hospital in Sydney and at St Vincent's Hospital in Melbourne, and transplants are performed at these centres and at the Royal Adelaide Hospital. Islet transplants are offered to those who have life-threatening hypoglycaemia. The results have been impressive, with reductions in both insulin requirement and hypoglycaemia; $44 \%$ of recipients still did not require insulin 3 years after transplant (usually an endovascular procedure, not a surgical procedure). These benefits come, again, at the expense of lifelong immunosuppression and its attendant risks. ${ }^{32}$ Limited tissue supply is a key barrier to more widespread use of islet transplantation. Advances in stem-cell technologies or in the production of porcine islets for human transplant may overcome this problem in the future.

\section{Prognosis in T1D}

Despite recent advances, T1D is still associated with considerable premature mortality caused by acute and chronic complications, particularly ischaemic heart disease. $^{33}$ The presence and severity of chronic kidney disease and ischaemic heart disease predict all-cause mortality in T1D. ${ }^{34,35}$ Recent reports of improved life expectancy in the $\mathrm{US}^{36}$ and in Denmark ${ }^{37}$ nonetheless provide great hope for persons with T1D and their clinicians.

Competing interests: No relevant disclosures.

Provenance: Commissioned; externally peer-reviewed. n

(C) 2015 AMPCo Pty Ltd. Produced with Elsevier B.V. All rights reserved.

References are available online at www.mja.com.au. 
1 Australian Institute of Health and Welfare. How common is diabetes? [website]. 2015. http://www.aihw.gov.au/howcommon-is-diabetes (accessed Jul 2015).

2 Skyler JS, Ricordi C. Stopping type 1 diabetes: attempts to prevent or cure type 1 diabetes in man. Diabetes 2011; 60: 1-8.

3 Catanzariti L, Faulks K, Moon L, et al. Australia's national trends in the incidence of type 1 diabetes in 0-14-year-olds, 2000-2006. Diabet Med 2009; 26: 596-601.

4 Wentworth JM, Fourlanos S, Harrison LC. Reappraising the stereotypes of diabetes in the modern diabetogenic environment. Nat Rev Endocrinol 2009; 5: 483-489.

5 Monami M, Marchionni N, Mannucci E. Long-acting insulin analogues vs. NPH human insulin in type 1 diabetes. A metaanalysis. Diabetes Obes Metab 2009; 11: 372-378.

6 Heller S, Buse J, Fisher M, et al. Insulin degludec, an ultralongacting basal insulin, versus insulin glargine in basal-bolus treatment with mealtime insulin aspart in type 1 diabetes (BEGIN Basal-Bolus Type 1): a phase 3, randomised, openlabel, treat-to-target non-inferiority trial. Lancet 2012; 379: 1489-1497.

7 Schmidt S, Schelde B, Norgaard K. Effects of advanced carbohydrate counting in patients with Type 1 diabetes: a systematic review. Diabet Med 2014; 31: 886-896.

8 McIntyre HD. Dose adjustment for normal eating: a role for the expert patient? Diabetes Metab J 2014; 38: 87-91.

9 Frier BM. Hypoglycaemia in diabetes mellitus: epidemiology and clinical implications. Nat Rev Endocrinol 2014; 10: 711-722.

10 Hendrieckx C, Halliday JA, Bowden JP, et al. Severe hypoglycaemia and its association with psychological wellbeing in Australian adults with type 1 diabetes attending specialist tertiary clinics. Diabetes Res Clin Pract 2014; 103: 430-436.

11 O'Connell SM, Cooper MN, Bulsara MK, et al. Reducing rates of severe hypoglycemia in a population-based cohort of children and adolescents with type 1 diabetes over the decade 2000-2009. Diabetes Care 2011; 34: 2379-2380.

12 Schmidt S, Meldgaard M, Serifovski N, et al. Use of an automated bolus calculator in MDI-treated type 1 diabetes: the BolusCal Study, a randomized controlled pilot study. Diabetes Care 2012; 35: 984-990.

$13 \mathrm{Xu}$ S, Alexander K, Bryant W, et al. Healthcare professional requirements for the care of adult diabetes patients managed with insulin pumps in Australia. Intern Med J 2015; 45: 86-93.

14 Misso ML, Egberts KJ, Page M, et al. Continuous subcutaneous insulin infusion (CSII) versus multiple insulin injections for type 1 diabetes mellitus. Cochrane Database Syst Rev 2010: CD005103.

15 Pickup JC, Freeman SC, Sutton AJ. Glycaemic control in type 1 diabetes during real time continuous glucose monitoring compared with self monitoring of blood glucose: metaanalysis of randomised controlled trials using individual patient data. BMJ 2011; 343: d3805.

16 Ly TT, Nicholas JA, Retterath A, et al. Effect of sensoraugmented insulin pump therapy and automated insulin suspension vs standard insulin pump therapy on hypoglycemia in patients with type 1 diabetes: a randomized clinical trial. JAMA 2013; 310: 1240-1247.

17 Russell SJ, El-Khatib FH, Sinha M, et al. Outpatient glycemic control with a bionic pancreas in type 1 diabetes. $N$ Engl J Med 2014; 371: 313-325.
18 Redondo MJ, Foster NC, Libman IM, et al (2015). Prevalence of cardiovascular risk factors in youth with type 1 diabetes and elevated body mass index. Acta Diabetol Epub 2015 Jun 16. http://dx.doi.org/10.1007/s00592-015-0785-1.

19 Conway B, Miller RG, Costacou T, et al. Temporal patterns in overweight and obesity in type 1 diabetes. Diabet Med 2015; 27: 398-404.

20 Liu C, Wu D, Zheng X, et al. Efficacy and safety of metformin for patients with type 1 diabetes mellitus: a meta-analysis. Diabetes Technol Ther 2015; 17: 142-148.

21 Kielgast U, Krarup T, Holst JJ, Madsbad S. Four weeks of treatment with liraglutide reduces insulin dose without loss of glycemic control in type 1 diabetic patients with and without residual beta-cell function. Diabetes Care 2011; 34: 1463-1468.

22 Garg SK, Moser EG, Bode BW, et al. Effect of sitagliptin on post-prandial glucagon and GLP-1 levels in patients with type 1 diabetes: investigator-initiated, double-blind, randomized, placebo-controlled trial. Endocr Pract 2013; 19: 19-28.

23 Henry RR, Rosenstock J, Edelman S, et al. Exploring the potential of the SGLT2 inhibitor dapagliflozin in type 1 diabetes: a randomized, double-blind, placebo-controlled pilot study. Diabetes Care 2015; 38: 412-419.

24 The effect of intensive treatment of diabetes on the development and progression of long-term complications in insulin-dependent diabetes mellitus. The Diabetes Control and Complications Trial Research Group. N Engl J Med 1993; 329: 977-986.

25 Cheung NW, Conn JJ, d'Emden MC, et al. Position statement of the Australian Diabetes Society: individualisation of glycated haemoglobin targets for adults with diabetes mellitus. Med J Aust 2009; 191: 339-344. https://www.mja.com.au/ journal/2009/191/6/position-statement-australian-diabetessociety-individualisation-glycated

26 Craig ME, Twigg SM, Donaghue KC, et al., for the Australian Type 1 Diabetes Guidelines Expert Advisory Group. National evidence-based clinical care guidelines for type 1 diabetes in children, adolescents and adults. Canberra: Australian Government Department of Health and Ageing, 2011. https:// diabetessociety.com.au/documents/Typelguidelines14Nov2011. pdf (accessed Jun 2015).

27 Bell R, Glinianaia SV, Tennant PW, et al. Peri-conception hyperglycaemia and nephropathy are associated with risk of congenital anomaly in women with pre-existing diabetes: a population-based cohort study. Diabetologia 2012; 55: 936-947.

28 Australian Institute of Health and Welfare. Diabetes in pregnancy: its impact on Australian women and their babies. Canberra: AlHW, 2010. (Cat. No. CVD 52; Diabetes Series No. 14.) http://www.aihw.gov.au/publication-detail/? $i d=6442472448$ (accessed Jul 2015).

29 Murphy HR. Integrating educational and technological interventions to improve pregnancy outcomes in women with diabetes. Diabetes Obes Metab 2010; 12: 97-104.

30 Penno MA, Couper JJ, Craig ME, et al. Environmental determinants of islet autoimmunity (ENDIA): a pregnancy to early life cohort study in children at-risk of type 1 diabetes. BMC Pediatr 2013; 13: 124.

31 Gruessner RW, Gruessner AC. The current state of pancreas transplantation. Nat Rev Endocrinol 2013; 9: 555-562. 
32 Bruni A, Gala-Lopez B, Pepper AR, et al. Islet cell transplantation for the treatment of type 1 diabetes: recent advances and future challenges. Diabetes Metab Syndr Obes 2014; 7: 211-223.

33 Livingstone SJ, Levin D, Looker $\mathrm{HC}$, et al. Estimated life expectancy in a Scottish cohort with type 1 diabetes, 2008-2010. JAMA 2015; 313: 37-44.

34 Groop PH, Thomas MC, Moran JL, et al. The presence and severity of chronic kidney disease predicts all-cause mortality in type 1 diabetes. Diabetes 2009; 58: 1651-1658.
35 Writing Group for the DERG, Orchard TJ, Nathan DM, et al. Association between 7 years of intensive treatment of type 1 diabetes and long-term mortality. JAMA 2015; 313: 45-53.

36 Miller RG, Secrest AM, Sharma RK, et al. Improvements in the life expectancy of type 1 diabetes: the Pittsburgh Epidemiology of Diabetes Complications study cohort. Diabetes 2012; 61: 2987-2992.

37 Jorgensen ME, Almdal TP, Carstensen B. Time trends in mortality rates in type 1 diabetes from 2002 to 2011. Diabetologia 2013; 56: 2401-2404. 